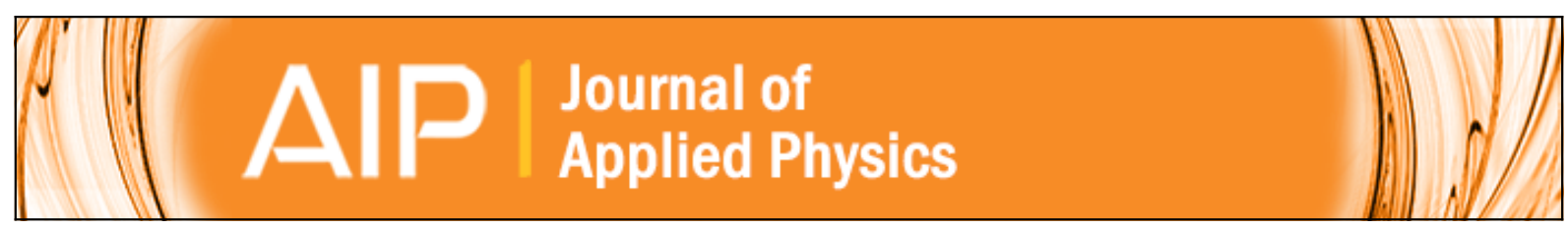

\title{
Transparent metal electrodes from ordered nanosphere arrays
}

Anthony J. Morfa, Eser M. Akinoglu, Jegadesan Subbiah, Michael Giersig, and Paul Mulvaney

Citation: Journal of Applied Physics 114, 054502 (2013); doi: 10.1063/1.4816790

View online: http://dx.doi.org/10.1063/1.4816790

View Table of Contents: http://scitation.aip.org/content/aip/journal/jap/114/5?ver=pdfcov

Published by the AIP Publishing

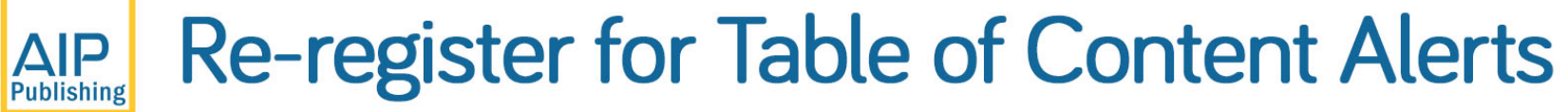

Create a profile.

Sign up today! 


\title{
Transparent metal electrodes from ordered nanosphere arrays
}

\author{
Anthony J. Morfa, ${ }^{1,2}$ Eser M. Akinoglu, ${ }^{2}$ Jegadesan Subbiah, ${ }^{1}$ Michael Giersig, ${ }^{2}$ \\ and Paul Mulvaney ${ }^{1}$ \\ ${ }^{1}$ School of Chemistry and Bio21 Institute, University of Melbourne, Parkville, Victoria 3010, Australia \\ ${ }^{2}$ Institut fur Experimentalphysik, Freie Universität Berlin, Arnimallee, 1414195 Berlin, Germany
}

(Received 2 May 2013; accepted 12 July 2013; published online 2 August 2013)

\begin{abstract}
We show that perforated metal electrode arrays, fabricated using nanosphere lithography, provide a viable alternative to conductive metal oxides as transparent electrode materials. The inter-aperture spacing is tuned by varying etching times in an oxygen plasma, and the effect of inter-aperture "wire" thickness on the optical and electronic properties of perforated silver films is shown. Optical transmission is limited by reflection and surface plasmons, and for these results do not exceed $73 \%$. Electrical sheet resistance is shown to be as low as $3 \Omega \square^{-1}$ for thermally evaporated silver films. The performance of organic photovoltaic devices comprised of a P3HT:PCBM bulk heterojunction deposited onto perforated metal arrays is shown to be limited by optical transmission, and a simple model is presented to overcome these limitations. (C) 2013 AIP Publishing LLC.

[http://dx.doi.org/10.1063/1.4816790]
\end{abstract}

\section{INTRODUCTION}

Transparent electrodes, most often transparent conducting oxides, are critical for high performance optoelectronic devices such as thin-film photovoltaic cells and light emitting diodes. The most common transparent conductor is indium tin oxide (ITO). However, the limited global supply of indium has begun to drive research into alternative transparent conductors. ${ }^{1}$ Several other metal oxides have achieved high optical transmission while maintaining low sheet resistance, ${ }^{2}$ including fluorine tin oxide (FTO) and aluminum zinc oxide (AZO). ${ }^{1-3}$ Nevertheless, a variety of other materials and structured films also exhibit high electrical conductivity including graphene, ${ }^{3-5}$ carbon nanotube meshes, ${ }^{3,6,7}$ metal nanowire meshes, ${ }^{3,8,9}$ and aperture-perforated metal films. ${ }^{10-13}$ Comparative performance of these materials has recently been reviewed by Barnes et al. ${ }^{3}$ using different figures of merit, such as the Haacke number. ${ }^{14}$

Transparent metal electrodes, with optical transmission achieved through apertures or holes, can conceivably achieve very high optical transmission while maintaining the low sheet resistance of the metal used. ${ }^{1}$ Kang et al. ${ }^{11,12,15}$ have demonstrated impressive lithography-based results with this approach. Such electrodes require one mask for each electrode geometry, while a more general approach using solution processed nano- or micro-spheres results in a tunable system to make similar, albeit round, apertures. Such masks can be made using conventional nanosphere techniques, ${ }^{16-20}$ random sphere geometries, ${ }^{13,21}$ or convective assembly. ${ }^{22}$ To date, microsphere based electrodes have been limited in transparency ${ }^{10,23}$ primarily because the goal of these investigations has been to engineer plasmonic resonances, not to maximize the DC conductivity of the film. ${ }^{13,24}$

For transparent metal electrodes to compete with transparent conductive oxides (TCOs), higher transmission must be achieved while maintaining low sheet resistances. In this work, solution-based, highly ordered apertures are studied in thin silver films and compared with other transparent metal electrodes. Post-processing is used to vary the inter-aperture "wire" thickness from 60 to $180 \mathrm{~nm}$. The effect of interaperture "wire" thickness on optical transmission and conductivity is investigated, and sheet resistances as low as $3 \Omega \square^{-1}$ are demonstrated with an average percent transmission as high as $70 \%$. A simple model is presented to calculate the transmittivity, which predicts that the transmission may reach values as high as $82 \%$. Thin-film organic photovoltaic devices are produced using these electrodes, and we show that the values of the key photovoltaic parameters: $\mathrm{V}_{\mathrm{oc}}$, fill factor (FF), and series resistance are all maintained with these electrodes, i.e., the photocurrent decreases proportionally to the absorption of the electrode. The results of various metal-aperture electrode structures are reviewed, and the ultimate limitations are discussed.

\section{RESULTS AND DISCUSSION}

Large-area, reproducible, and tunable electrodes were fabricated using nanosphere lithography to generate the mask, followed by reactive ion etching and finally metal evaporation. First, $756 \mathrm{~nm}$ diameter polystyrene (PS) spheres were ordered using traditional nanosphere lithography, bound to a glass surface by heating the glass/PS system to $105^{\circ} \mathrm{C}$, just over the glass transition point of PS, for $\sim 3 \mathrm{~s}$. The diameter of the PS spheres was then reduced in oxygen plasma (see schematic of this process in Figure 1(a) and actual SEM micrographs of etched spheres in Figures 1(b)-1(d)). Lastly, a rectangular bottom electrode, approximately $7 \mathrm{~mm}$ wide, was masked using Kapton tape, through which a $3 \mathrm{~nm}$ titanium layer followed by $50 \mathrm{~nm}$ of silver was evaporated. Finally, the Kapton tape was removed physically, and the remaining PS spheres were dissolved in toluene.

The result is a silver film with an ordered array of apertures of varying diameter (see Figure 2). For a fixed initial sphere size (in our case $756 \mathrm{~nm}$ ), the aperture diameter and inter-aperture "wire" width can be tuned by varying etching times. As expected, films with very low aperture coverage 


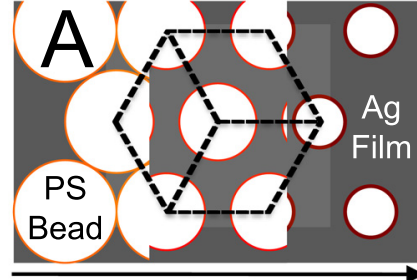

Increasing Etch Time

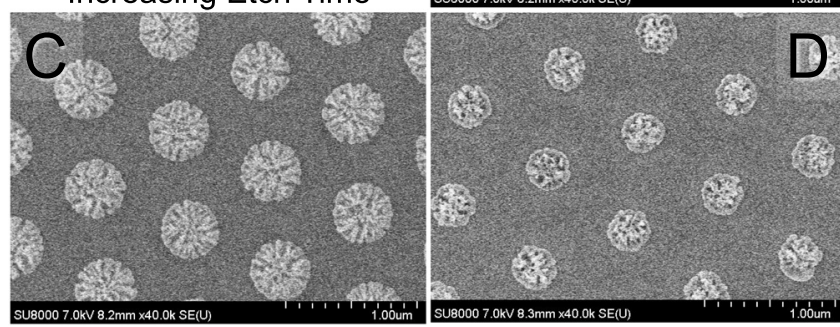

FIG. 1. (A) 2D diagram of inter-particle area after PS sphere mask has been etched in oxygen plasma. Sphere (white circles) diameter decreases with increasing etch time (as you move right in diagram), resulting in more intersphere area or silver film coverage. (B) SEM micrographs of hexagonal arrays of PS spheres (average initial diameter $=764 \mathrm{~nm}$ ) on silicon-before etching; (C) after etching in oxygen plasma for $400 \mathrm{~s}$ with an average diameter of $479 \mathrm{~nm}$; (D) after etching in oxygen plasma for $600 \mathrm{~s}$ with an average diameter of $350 \mathrm{~nm}$.

have low optical transmission (see Figure 3). As the etch time in oxygen plasma is increased, the aperture diameter decreases, leaving, after metal deposition, a larger interaperture metal "wire." As can be seen from Figures 2(a) to $2(d)$, the "wire" thickness increases from 60 to $180 \mathrm{~nm}$ at etching times between $250 \mathrm{~s}$ and $600 \mathrm{~s}$.

Likewise, the optical and electronic properties of the resulting silver film vary greatly as the apertures are decreased (see Figure 3). As the aperture diameter is decreased, the resulting percent transmission (see Figure 3(a)) decreases due to reduced aperture coverage and an increasing inter-aperture "wire" thickness (see Figure 3(b)). The average percent transmission can be predicted using a simple geometric model

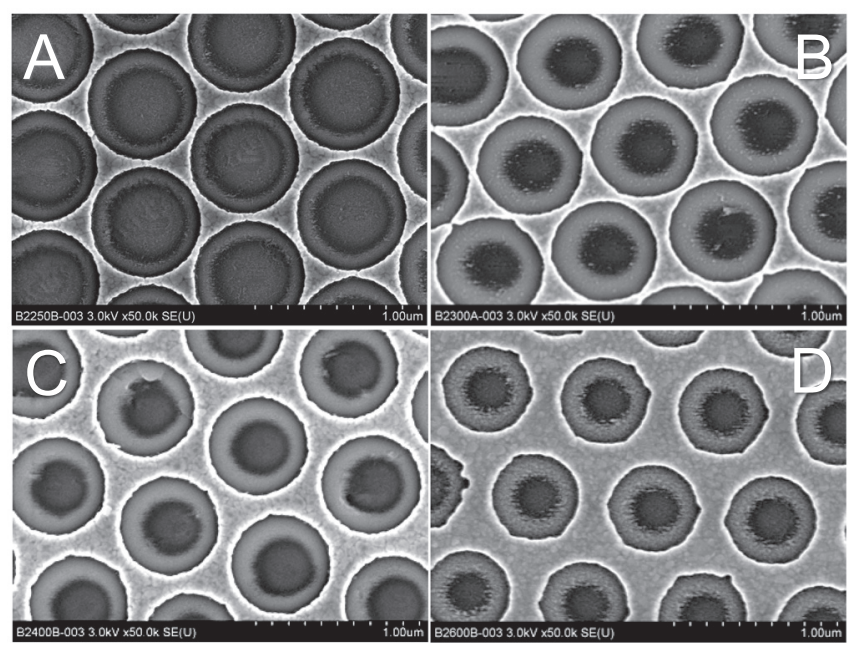

FIG. 2. SEM micrographs showing the individual metal films evaporated through PS sphere masks (initial diameter $=756 \mathrm{~nm}$ ) where the dark "holes" are glass and the gray hexagonal lattice is a $3 \mathrm{~nm}$ titanium layer covered with $50 \mathrm{~nm}$ thick layer of silver. The original sphere mask was etched for (a) $250 \mathrm{~s}$ with a final aperture diameter of $695 \mathrm{~nm}$; (b) $300 \mathrm{~s}$ with final aperture diameter of $685 \mathrm{~nm}$; (c) $400 \mathrm{~s}$ with final aperture of $635 \mathrm{~nm}$; (d) and $600 \mathrm{~s}$ with a final aperture diameter of $575 \mathrm{~nm}$. whereby the 2D surface area of the apertures is assumed to transmit $100 \%$ of the incident light and the 2D surface area of the metal "wires" and interstitial areas reflect $100 \%$ of the incident light. Ignoring surface plasmon absorption or imperfections in the 2D ordering (for a theoretical consideration of the plasmonic contribution, see the work of Peng et al. ${ }^{25}$ ), the percent transmission follows Eq. (1)

$$
T=100 \% \cdot \frac{\pi \cdot\left(r_{o}-\frac{t}{2}\right)^{2}}{2 \sqrt{3} \cdot r_{o}^{2}},
$$

where $r_{o}$ is the initial sphere radius and $t$ is the inter-aperture "wire" thickness. For films where the "wire" thickness is $61 \mathrm{~nm}$ (etched $250 \mathrm{~s}$ ), this model predicts the average percent transmission (over the spectral region from 350-1200 nm) to within $\approx 5 \%$ ( $77 \%$ predicted versus $73 \%$ measured), whereas, for "wire" thicknesses between 120 and $180 \mathrm{~nm}$ (etched 500 and $600 \mathrm{~s}$, respectively), the model predicts the percent transmission to within $\approx 20 \%$ (53\% predicted versus $43 \%$ actual). This increase in the deviation results from the increased surface plasmon absorption in both the visible $(650 \mathrm{~nm})$ and the infrared (IR) $(950 \mathrm{~nm})$ which can be clearly seen in Figure 3(a).

An increased wire thickness leads to a decrease in sheet resistance of the film (see Figure 3(c)). At very small wire thicknesses the sheet resistance is substantially larger than the values obtained with other transparent metal electrodes (see Table I). For samples etched for 250 s, the wire thickness is, on average, $61 \pm 18 \mathrm{~nm}$ with a sheet resistance of $136 \Omega \square^{-1}$. A significant fraction (over 15\%) of the wires are thinner than the electron mean free path in silver $(520 \AA),{ }^{26}$ which greatly increases the resistance of these wires. As the wire thickness increases to over $100 \mathrm{~nm}$, the sheet resistance of the electrode is approximately $10 \Omega \square^{-1}$ and approaches that obtained with electrodes that are composed of a randomly placed $30 \%$ coverage of apertures (compared to $64 \%$ herein). ${ }^{27}$ As the wire thickness approaches $200 \mathrm{~nm}$, the sheet resistance is below $3 \Omega \square^{-1}$, which is lower than other reported transparent conductors and only a factor of three higher than similarly produced metals films without apertures. ${ }^{27}$ Although low sheet resistances are preferable, when fabricating a thin-film optoelectronic device, both low sheet resistances and high optical transmission must be achieved.

TABLE I. "Haacke" number (Eq. (2)) for several metal-aperture-based transparent conducting electrodes with ITO for reference. The value of 13.9 in the NSL row is the predicted value for a film with $1700 \mathrm{~nm}$ spheres, etched to a $120 \mathrm{~nm}$ "wire"; the $\% \mathrm{~T}$ is predicted to be $78 \%$ and the $\mathrm{R}_{\mathrm{S}}$ value is taken from the data in this study $\left(6 \Omega \square^{-1}\right)$. The data marked $\ddagger, \dagger, \diamond$ are based on the average optical transmission between 400 and $800 \mathrm{~nm}$, from Refs. 10-12, respectively.

\begin{tabular}{|c|c|c|c|}
\hline & $\mathrm{R}_{\mathrm{s}}\left(\Omega \square^{-1}\right)$ & $\% \mathrm{~T}^{(300-1200 \mathrm{~nm})}$ & $\phi_{\mathrm{H}}$ \\
\hline $\mathrm{ITO}^{3}$ & 12.5 & 86.3 & 19.2 \\
\hline $\mathrm{Ag} \mathrm{NW}$ & 23 & 81.1 & 5.3 \\
\hline Au Grid ${ }^{12}$ & 8 & $77^{\diamond}$ & $9.2^{\diamond}$ \\
\hline Ag Grid ${ }^{11}$ & 4.8 & $68.3^{\dagger}$ & $4.6^{\dagger}$ \\
\hline $\mathrm{Au} \mathrm{NSL}^{10}$ & 6.2 & $65^{\ddagger}$ & $2.2^{\ddagger}$ \\
\hline $\mathrm{Ag} \mathrm{NSL}^{(400 s)}$ & 6.1 & 62.1 & $1.4(13.9)$ \\
\hline $\mathrm{Ag} \mathrm{NSL}^{(600 s)}$ & 2.5 & 43.3 & 0.094 \\
\hline
\end{tabular}



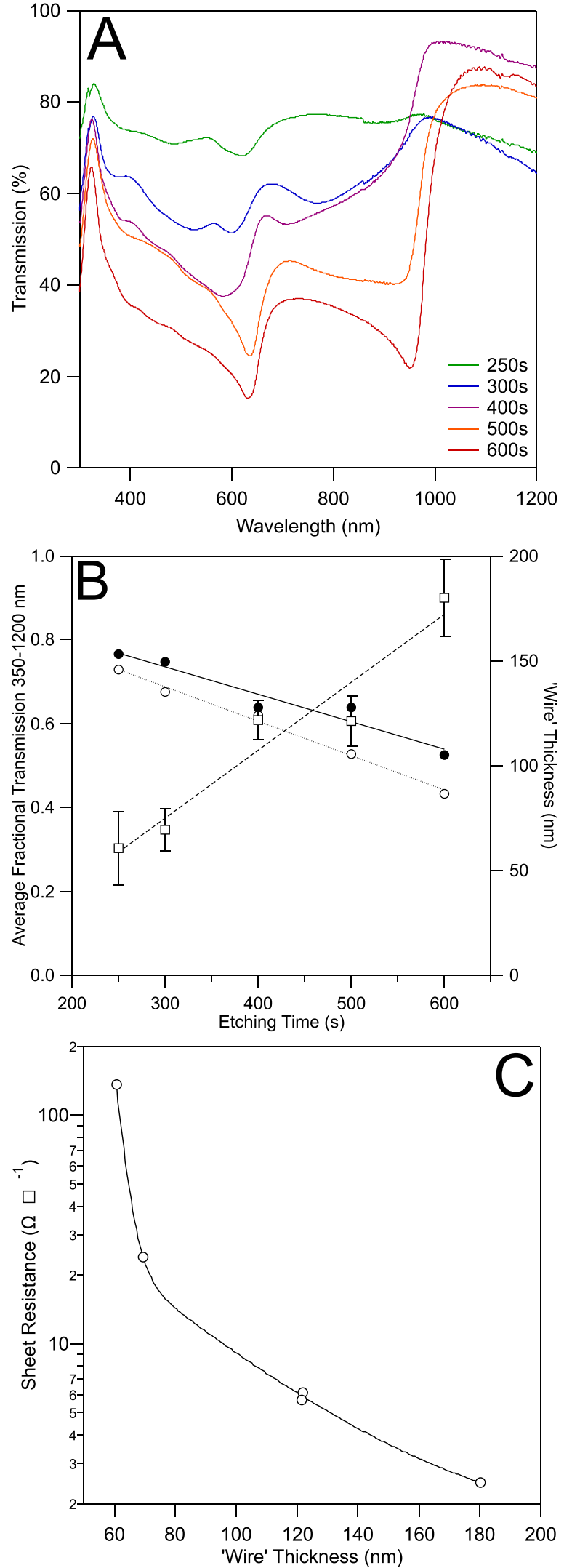

FIG. 3. (a) As the aperture diameter decreases the transmittivity decreases (a strong absorbance due to surface plasmon excitation ${ }^{30}$ is seen $\sim 950 \mathrm{~nm}$ and Wood's anomaly ${ }^{31,32}$ is seen at $\sim 650 \mathrm{~nm}$ ). (b) The measured (open circles) and predicted (using Eq. (1), filled circles) average percent transmission (from 400 to $1200 \mathrm{~nm}$, left axis) decrease linearly while the "wire" thickness (right axis) increases linearly with increasing etch time. (c) The sheet resistance measured using four-point probe as a function of the measured "wire" thickness.
In Figure 4(a), we compare current-voltage measurements collected from organic photovoltaic devices fabricated using both nanosphere metal electrodes and ITO. The standard P3HT:PCBM organic bulk heterojunction was chosen for these comparisons. As was demonstrated by Kang et al., ${ }^{12}$ for nanowire electrodes, the spacing between wires must be minimized when the materials used to produce a photovoltaic device have high sheet resistances. For the electrodes produced for this work, with a period of $756 \mathrm{~nm}$, and a PEDOT:PSS sheet resistance of $150 \mathrm{k} \Omega \square^{-1},{ }^{12}$ the expected voltage drop from resistive losses is expected to be below $10 \mathrm{mV} .{ }^{12}$ This is in fact the case here; when the electrodes were incorporated into a standard organic photovoltaic device structure, the photovoltaic efficiency was affected largely by optical transmission (see Figure 4(a)), but the $\mathrm{V}_{\mathrm{OC}}$ only decreases slightly (from $0.58 \mathrm{~V}$ with an ITO electrode to $0.57 \mathrm{~V}$ with the silver electrode) (see Figure 4(b)).

Although the electrodes with the thinnest wires transmit the most light, they have the highest sheet resistance, and as such they are greatly hampered by resistive losses in the complete device. As the wire thickness is increased from 60 to $180 \mathrm{~nm}$, the series resistance, calculated at $1 \mathrm{~V}$, decreases from 416 to $46 \Omega \mathrm{cm}^{2}$, compared to $29 \Omega \mathrm{cm}^{2}$ for the device made on ITO (see Figure 4(c)). The ultimate limitation on device performance, as the wire thickness increases, is optical transmission. The transparent metal electrode (TME) devices with large wire thicknesses $(120 \mathrm{~nm}$ and above) reach a power conversion efficiency of $1.3 \%$, compared to $2.9 \%$ on ITO, i.e., $45 \%$ of the reference. Optically, for devices with the same wire thicknesses, the TMEs only transmit $50 \%$ as much light as ITO. This demonstrates a non-intuitive response; initially, the largest detriment to device performance is resistive losses, seen in the high initial series resistance, but once the sheet resistance of the silver mesh increases, the reflective losses are the limiting factor. Though transparent metal electrodes are often reported to produce "plasmon enhancements," the optical properties of these electrodes simply scale with aperture surface area (see Figure 4(d)). With thicker wires than those used here, the optical transmission would only decrease further, with limited gain in conductivity (for wire thickness above $120 \mathrm{~nm}$, the electrodes have a sheet resistance below that of ITO $\left.\left(10 \Omega \square^{-1}\right)\right)$.

To understand the limitation of optical transmission on the effectiveness of these electrodes, we employ the Haacke $^{14}$ number (see Eq. (2)), as discussed by Barnes et $a .^{3}$ In that work different transparent conducting media were compared to ITO. For this work, metal-based transparent electrodes are most applicable, and in Table I, we show how the optical transmission compares across several silverand gold-based electrodes as well as ITO. The sheet resistance of the electrode and the Haacke number are also presented. By far, the best transparent electrode in this list is still ITO. However, it is clear that lithographically produced metal electrodes have much lower sheet resistances than ITO or silver nanowire meshes. Indeed the NSL sample from this study etched for $600 \mathrm{~s}$ has the lowest sheet resistance of all the samples. The main disadvantage of metal-based systems can be clearly seen when one compares the $\% \mathrm{~T}$ of all of the 

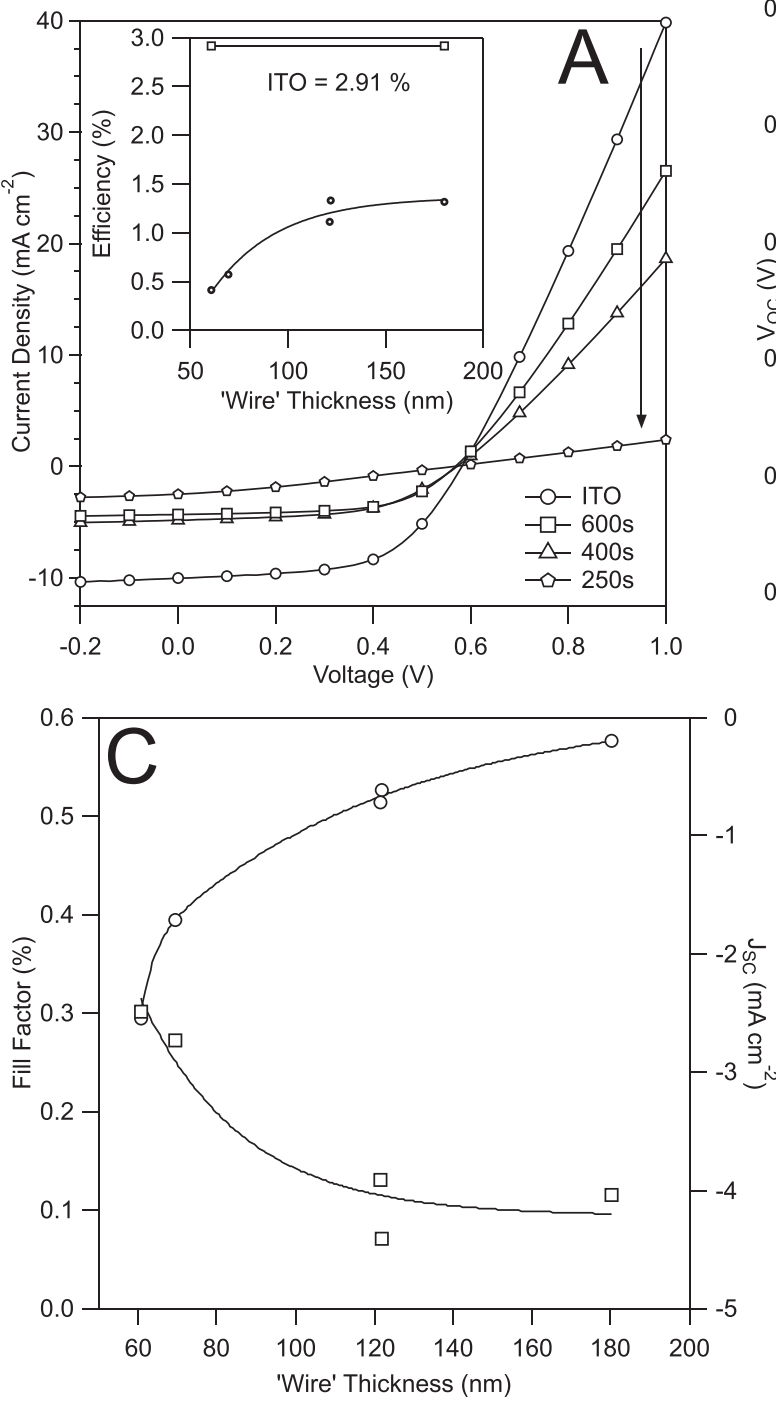
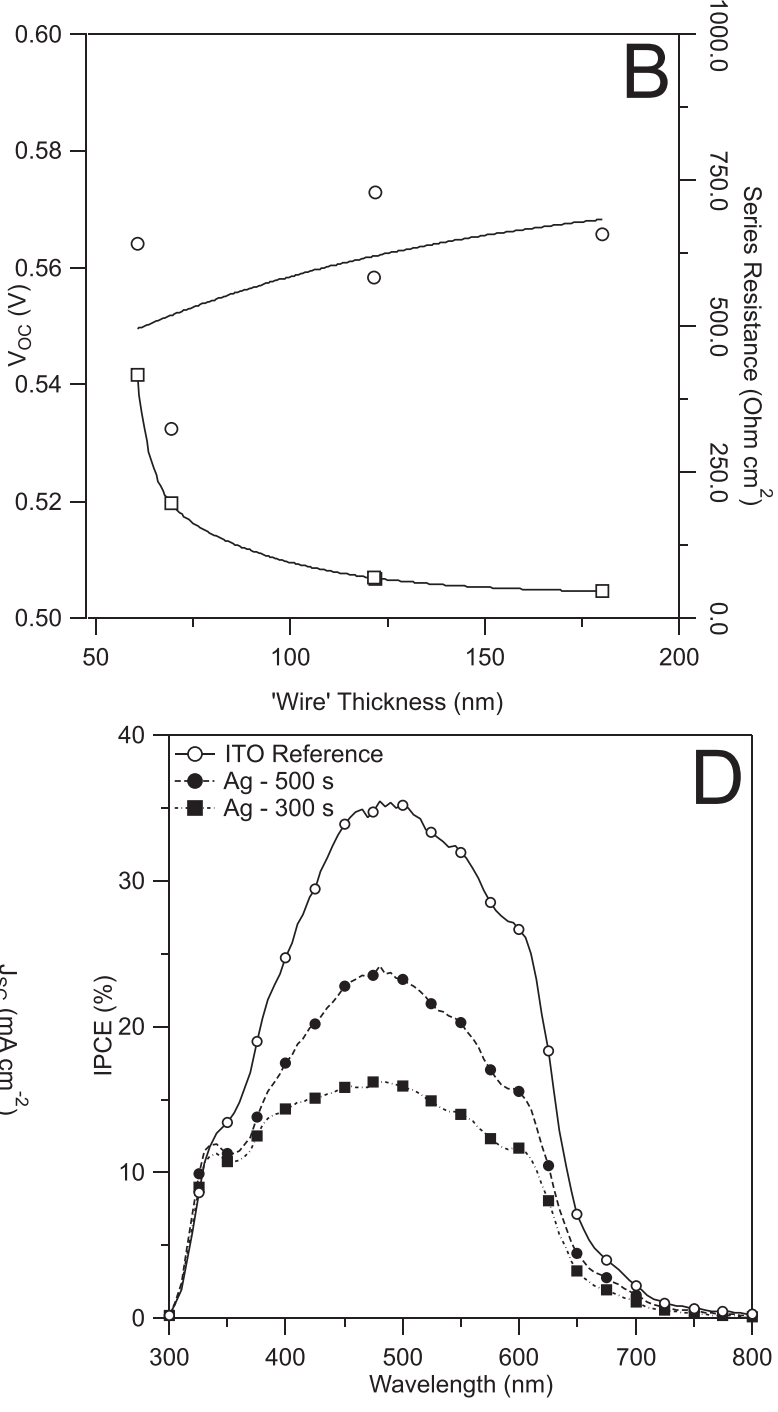

FIG. 4. (a) Photovoltaic photocurrent-voltage curves under AM 1.5G illumination for representative silver aperture electrodes and ITO (the inset shows determined maximum power conversion efficiency versus "wire" thickness). (b) V $\mathrm{OC}_{\mathrm{OC}}$ and series resistance (left and right axis, respectively) and (c) fill factor and short-circuit current density (left and right axis, respectively) versus the measured "wire" thickness of the electrodes. (d) Measured optical response of the devices across visible wavelengths.

materials. While ITO and Ag nanowire meshes have average $\% \mathrm{~T}$ values (from 300 to $1200 \mathrm{~nm}$ ) above $80 \%$, each of the lithographically produced samples has an average percent transmission below $80 \%$ (where the data from Ho et al., marked " $\ddagger, " 10$ and Guo and coworkers, marked " $\dagger "$ "11 or ",, 12 are only averaged from 400 to 800 ). The overall effect of the low transmittivity is to limit the Haacke number below about 5

$$
\phi_{\mathrm{H}}=(\% \mathrm{~T})^{10} / R_{\square} .
$$

Of course, the average percent transmission can be greatly improved if the original sphere, or aperture diameter, is increased. Unfortunately, for low mobility materials such as common organic semiconductors, increasing the aperture diameter will cause resistive losses over $10 \mathrm{mV} .^{12}$ If for example an electrode is fabricated from $1700 \mathrm{~nm}$ diameter spheres with a $120 \mathrm{~nm}$ "wire" between the resulting apertures, the percent transmission from Eq. (1) would be $78 \%$ and, assuming the sheet resistance is similar to that found in this study $\left(2.5-6 \Omega \square^{-1}\right)$, the Haacke number would be between 13.9 and 33.3. (Of course, hexagonal-close-packed circular apertures are less space efficient than square apertures, which would have even higher percent transmissions). For this aperture diameter, different photovoltaic materials should be used, because the limitation identified by Karng et $a .^{12}$ is largely a result of the low conductivity of PEDOT:PSS and could be overcome with different material selection such as $\mathrm{ZnO}$ (in the well-known inverted organic photovoltaic device structure).

In conclusion, transparent metal electrodes have been produced utilizing a solution-processed, highly ordered array of nano-apertures in silver. The inter-aperture separation or "wire" thickness was varied between 60 and $180 \mathrm{~nm}$, leading to controllable optical and electronic properties. Optical transmission decreased from $73 \%$ to $43 \%$ as wire thickness increased, and sheet resistance decreased from $120 \Omega \square^{-1}$ to $3 \Omega \square^{-1}$ for the thickest "wires." These electrodes were found to have a lower electrical resistivity than other nano-aperture based electrodes, but also lower optical transmission. A simple 
geometric model for optical transmission is presented that predicts improved transmission for larger aperture diameters with a corresponding increase in these films' Haacke number. With recent advances in the preparation of large scale, highly ordered polystyrene sphere arrays, these electrodes present an excellent alternative to transparent conducting oxides and lithographically produced metal electrodes.

\section{EXPERIMENTAL}

\section{Nanosphere arrays}

$756 \mathrm{~nm}$ polystyrene beads ( $8 \%$ by weight in water), purchased from Microparticles $\mathrm{GmbH}$ Berlin, used after diluting 1:2 in ethanol, were slowly dispersed onto a water (18 M $\Omega$-MilliQ) surface. Once a large array was assembled and ordered, the water was removed through pumping and evaporation, causing the array to be placed on a cleaned float glass surface. ${ }^{28}$ The glass was cleaned by sonication: first in acetone and then isopropanol for $15 \mathrm{~min}$ each. The glass was then placed on a hot plate, with a surface temperature of $105^{\circ} \mathrm{C}$, for $\sim 3 \mathrm{~s}$. The spheres were etched in an oxygen plasma at a pressure of $0.08 \mathrm{mbars}$ and power of $64 \mathrm{~W}$ for times varying between 250 and $600 \mathrm{~s}$ in a Plasma Technology MiniFlecto system. Lastly, a $7 \mathrm{~mm}$ wide strip was masked using Kapton tape, and $3 \mathrm{~nm}$ of titanium at $0.2 \mathrm{~nm} \mathrm{~s}^{-1}$ followed by $50 \mathrm{~nm}$ of silver at $0.3 \mathrm{~nm} \mathrm{~s}^{-1}$, was evaporated in a custombuilt e-beam evaporator. The Kapton tape was removed and the entire system cleaned in toluene, which dissolves the spheres.

\section{Solar cell preparation}

On to cleaned ITO or silver mesh electrodes, PEDOT:PSS (Baytron AI 4083 from HC Starck) was spun at $6000 \mathrm{rpm}$ for $30 \mathrm{~s}$, followed by a 1:1 blend (by weight ratio)) of P3HT (Merck) and $\mathrm{PC}_{60} \mathrm{BM}$ (purchased from solenne $\mathrm{BV}$ ) in ortho-dichlorobenzene at a total solute concentration of $20 \mathrm{mg} \mathrm{ml}^{-1}$ at $800 \mathrm{rpm}$ for $60 \mathrm{~s}$. Once the P3HT:PC ${ }_{60} \mathrm{BM}$ layer was dry, a $\mathrm{ZnO}$ nanoparticle film was spun from $5 \mathrm{~nm}$ $\mathrm{ZnO}$ nanoparticles, in ethanol solution, ${ }^{29}$ at a total concentration of $25 \mathrm{mg} \mathrm{ml}^{-1}$ at a spin speed of $3000 \mathrm{rpm}$ for $30 \mathrm{~s}$. Lastly an aluminum electrode was thermally evaporated at a rate for $0.2 \AA^{-1}$ for the first $10 \mathrm{~nm}$ and then $2 \AA \mathrm{s}^{-1}$ until the total metal film thickness was $100 \mathrm{~nm}$ through a shadow mask at approximately $1 \times 10^{-6}$ Torr.

\section{Film characterization}

SEM characterization of PS beads and silver films was made on a Hitachi SU8030 SEM. Data from Figure 1 were of samples on silicon with a native oxide. Four-point probe measurements were made using a Jandel electrode unit in the linear configuration with $635 \mu \mathrm{m}$ spacing and a load between 30 and $60 \mathrm{~g}$. Absorbance measurements were collected with a Perkin-Elmer Lambda 950 spectrophotometer, and photovoltaic characterization was measured using a Cary 2400 SMU with a Newport-Oriel solar simulator with AM 1.5G optical filtering for JV measurements or a Oriel 150W Xe lamp couple to an Oriel monochromator for incident photonto-current conversion efficiency (IPCE) measurements.

\section{ACKNOWLEDGMENTS}

The authors acknowledge Dr. Klaus Ellmer of HZB for silver evaporation and Dr. Bjorn Winther-Jensen of Monash University for access to a four-point probe. A.J.M. acknowledges financial support of the Alexander von Humboldt Stiftung. A.J.M. and E.M.A. acknowledge financial support of the BMBF under Projekt AUS 10/018. M.G. acknowledges financial support of H.Z.B. P.M. acknowledges the support of the Australian Academy of Science ISL program.

${ }^{1}$ D. S. Hecht, L. B. Hu, and G. Irvin, "Emerging transparent electrodes based on thin films of carbon nanotubes, graphene, and metallic nanostructures," Adv. Mater. 23(13), 1482-1513 (2011).

${ }^{2}$ H. Y. Liu, V. Avrutin, N. Izyumskaya, U. Ozgur, and H. Morkoc, "Transparent conducting oxides for electrode applications in light emitting and absorbing devices," Superlattices Microstruct. 48(5), 458-484 (2010). ${ }^{3}$ T. M. Barnes, M. O. Reese, J. D. Bergeson, B. A. Larsen, J. L. Blackburn, M. C. Beard, J. Bult, and J. van de Lagemaat, "Comparing the fundamental physics and device performance of transparent, conductive nanostructured networks with conventional transparent conducting oxides," Adv. Energy Mater. 2(3), 353-360 (2012).

${ }^{4}$ H. A. Becerril, J. Mao, Z. Liu, R. M. Stoltenberg, Z. Bao, and Y. Chen, "Evaluation of solution-processed reduced graphene oxide films as transparent conductors," ACS Nano 2(3), 463-470 (2008).

${ }^{5}$ G. Eda, G. Fanchini, and M. Chhowalla, "Large-area ultrathin films of reduced graphene oxide as a transparent and flexible electronic material," Nat. Nanotechnol. 3(5), 270-274 (2008).

${ }^{6}$ M. W. Rowell, M. A. Topinka, M. D. McGehee, H. J. Prall, G. Dennler, N. S. Sariciftci, L. B. Hu, and G. Gruner, "Organic solar cells with carbon nanotube network electrodes," Appl. Phys. Lett. 88(23), 233506 (2006).

${ }^{7}$ J. van de Lagemaat, T. M. Barnes, G. Rumbles, S. E. Shaheen, T. J. Coutts, C. Weeks, I. Levitsky, J. Peltola, and P. Glatkowski, "Organic solar cells with carbon nanotubes replacing $\mathrm{In}_{2} \mathrm{O}_{3}$ : $\mathrm{Sn}$ as the transparent electrode," Appl. Phys. Lett. 88(23), 233503 (2006).

${ }^{8}$ S. De, T. M. Higgins, P. E. Lyons, E. M. Doherty, P. N. Nirmalraj, W. J. Blau, J. J. Boland, and J. N. Coleman, "Silver nanowire networks as flexible, transparent, conducting films: Extremely high DC to optical conductivity ratios," ACS Nano 3(7), 1767-1774 (2009).

${ }^{9}$ J. Y. Lee, S. T. Connor, Y. Cui, and P. Peumans, "Solution-processed metal nanowire mesh transparent electrodes," Nano Lett. 8(2), 689-692 (2008).

${ }^{10}$ Y. H. Ho, K. Y. Chen, S. W. Liu, Y. T. Chang, D. W. Huang, and P. K. Wei, "Transparent and conductive metallic electrodes fabricated by using nanosphere lithography," Org. Electron. 12(6), 961-965 (2011).

${ }^{11}$ M. G. Kang and L. J. Guo, "Nanoimprinted semitransparent metal electrodes and their application in organic light-emitting diodes," Adv. Mater. 19(10), 1391 (2007).

${ }^{12}$ M. G. Kang, M. S. Kim, J. S. Kim, and L. J. Guo, "Organic solar cells using nanoimprinted transparent metal electrodes," Adv. Mater. 20(23), 4408-4413 (2008).

${ }^{13}$ T. H. Reilly, J. van de Lagemaat, R. C. Tenent, A. J. Morfa, and K. L. Rowlen, "Surface-plasmon enhanced transparent electrodes in organic photovoltaics," Appl. Phys. Lett. 92(24), 243304 (2008).

${ }^{14} \mathrm{G}$. Haacke, "New figure of merit for transparent conductors," J. Appl. Phys. 47(9), 4086-4089 (1976).

${ }^{15}$ M. G. Kang, T. Xu, H. J. Park, X. G. Luo, and L. J. Guo, "Efficiency enhancement of organic solar cells using transparent plasmonic Ag nanowire electrodes," Adv. Mater. 22(39), 4378 (2010).

${ }^{16}$ H. W. Deckman and J. H. Dunsmuir, "Natural lithography," Appl. Phys. Lett. 41(4), 377-379 (1982).

${ }^{17}$ N. D. Denkov, O. D. Velev, P. A. Kralchevsky, I. B. Ivanov, H. Yoshimura, and K. Nagayama, "Mechanism of formation of 2-dimensional crystals from latex-particles on substrates," Langmuir 8(12), 3183-3190 (1992).

${ }^{18} \mathrm{M}$. Giersig and P. Mulvaney, "Preparation of ordered colloid monolayers by electrophoretic deposition," Langmuir 9(12), 3408-3413 (1993).

${ }^{19}$ J. C. Hulteen and R. P. Vanduyne, "Nanosphere lithography - A materials general fabrication process for periodic particle array surfaces," J. Vac. Sci. Technol. A 13(3), 1553-1558 (1995).

${ }^{20}$ A. Kosiorek, W. Kandulski, P. Chudzinski, K. Kempa, and M. Giersig, "Shadow nanosphere lithography: Simulation and experiment," Nano Lett. 4(7), 1359-1363 (2004). 
${ }^{21}$ P. Hanarp, D. S. Sutherland, J. Gold, and B. Kasemo, "Control of nanoparticle film structure for colloidal lithography," Colloids Surf., A 214(1-3), 23-36 (2003).

${ }^{22}$ B. G. Prevo and O. D. Velev, "Controlled, rapid deposition of structured coatings from micro- and nanoparticle suspensions," Langmuir 20(6), 2099-2107 (2004).

${ }^{23}$ J. F. Zhu, X. D. Zhu, R. Hoekstra, L. Li, F. X. Xiu, M. Xue, B. Q. Zeng, and K. L. Wang, "Metallic nanomesh electrodes with controllable optical properties for organic solar cells," Appl. Phys. Lett. 100(14), 143109 (2012).

${ }^{24}$ W. A. Luhman, S. H. Lee, T. W. Johnson, R. J. Holmes, and S. H. Oh, "Self-assembled plasmonic electrodes for high-performance organic photovoltaic cells," Appl. Phys. Lett. 99(10), 103306 (2011).

${ }^{25}$ Y. Peng, T. Paudel, W. C. Chen, W. J. Padilla, Z. F. Ren, and K. Kempa, "Percolation and polaritonic effects in periodic planar nanostructures evolving from holes to islands," Appl. Phys. Lett. 97(4), 041901 (2010).

${ }^{26} \mathrm{~F}$. W. Reynolds and G. R. Stilwell, "Mean free paths of electrons in evaporated metal films," Phys. Rev. 88(2), 418-419 (1952).
${ }^{27}$ T. H. Reilly, R. C. Tenent, T. M. Barnes, K. L. Rowlen, and J. van de Lagemaat, "Controlling the optical properties of plasmonic disordered nanohole silver films," ACS Nano 4(2), 615-624 (2010).

${ }^{28}$ P. Patoka and M. Giersig, "Self-assembly of latex particles for the creation of nanostructures with tunable plasmonic properties," J. Mater. Chem. 21(42), 16783-16796 (2011).

${ }^{29}$ G. Sarasqueta, K. R. Choudhury, J. Subbiah, and F. So, "Organic and inorganic blocking layers for solution-processed colloidal PbSe nanocrystal infrared photodetectors," Adv. Funct. Mater. 21(1), 167-171 (2011).

${ }^{30}$ H. Raether, Surface-Plasmons on Smooth and Rough Surfaces and on Gratings (Springer-Verlag, 1988), Vol. 111, pp. 1-133.

${ }^{31} \mathrm{R}$. W. Wood XLII, "On a remarkable case of uneven distribution of light in a diffraction grating spectrum," Philos. Mag. Ser. 6 4(21), 396-402 (1902).

${ }^{32}$ A. Hessel and A. A. Oliner, "A new theory of Wood's anomalies on optical gratings,” Appl. Opt. 4(10), 1275 (1965). 